\title{
Hydro-Mechanical Coupling in Damaged Porous Media Containing Isolated Cracks or/and Vugs: Model and Computations
}

\author{
J. Lewandowska ${ }^{1}$ and J.-L. Auriault ${ }^{2}$ \\ ${ }^{1}$ Laboratory of Mechanics and Civil Engineering (LMGC), University Montpellier 2, \\ CC 048, Place Eugène Bataillon, 34095 Montpellier cedex 5, France, PH (+33) 467 \\ 1446 60; FAX (+33) 4671439 23; email: Jolanta.Lewandowska@univ-montp2.fr \\ ${ }^{2}$ Laboratory Soils, Solids, Structures - Risks (L3S-R), Joseph Fourier University, \\ email: jeanlouis.auriault@3sr-grenoble.fr
}

\begin{abstract}
In this paper we present the development of the macroscopic model describing the hydro-mechanical coupling of damaged porous media containing cracks or/and vugs, by using the asymptotic expansion method. The analysis starts at the mesoscopic scale at which we assume a generic microstructure and the validity of the Biot model in the micro-porous domain saturated by a fluid. In the crack/vug domain the Stokes equation is assumed. After estimation of orders of magnitude of different terms, the description is rendered non-dimensional and the homogenization process is carried out. It leads to an extended Biot model that possesses the same mathematical structure as the initial Biot model. However, the macroscopic poro-elasticity and the macroscopic Darcy conductivity are modified. In order to illustrate the performance of the model, numerical computations of a macroscopic boundary value problem were performed. The results show practical importance of modifications introduced in the Biot model.
\end{abstract}

\section{INTRODUCTION}

Natural or damaged geomaterials (soils, rocks or cement-based materials) contain very often cracks and/or vugs. In such cases the classical Biot model cannot be directly applied, since it was initially developed for single porosity homogeneous materials (Biot, 1941) In this paper we present the macroscopic model describing the hydro-mechanical behaviour of such class of geomaterials which was obtained by using the asymptotic expansion method. An elastic porous material with isolated cracks and/or vugs that are periodically distributed in the whole medium, is considered. The porous material is completely saturated by an incompressible fluid (example: water). 


\section{MESOSCOPIC PROBLEM}

The analysis starts at the mesoscopic scale (the crack/vug scale) at which we assume a generic microstructure, Figure 1, and the validity of the Biot model in the microporous domain (matrix) saturated by a fluid. In the crack/vug domain occupied exclusively by fluid, the Stokes equation is assumed. The description is completed by the continuity conditions on the boundary between the porous medium and the $\operatorname{crack}($ vug).

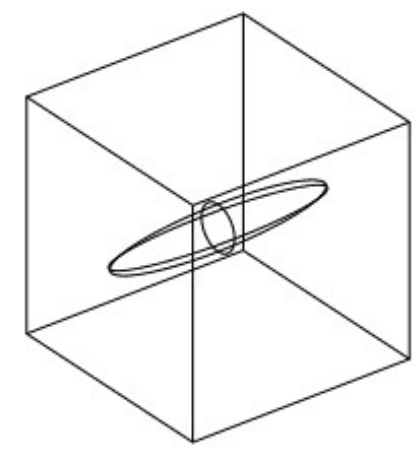

Figure 1 Microstructue of a porous medium with a crack (vug)

The dimensioless mesoscopic problem is formulated by Equations (1)-(11) (Lewandowska and Auriault, 2012):

In the microporous domain:

$$
\begin{aligned}
& \frac{\partial \sigma_{i j}^{m}}{\partial x_{j}}=0 \\
& \sigma_{i j}^{m}=\sigma_{i j}^{s}-\alpha p^{p} \\
& \sigma_{i j}^{s}=c_{i j k l} e_{x k l}\left(\boldsymbol{u}^{s}\right) \\
& \frac{\partial}{\partial x_{i}}\left(v^{p}-\phi^{p} \frac{\partial u_{i}^{s}}{\partial t}\right)=-\alpha \frac{\partial e_{x i i}\left(\boldsymbol{u}^{s}\right)}{\partial t}-\beta \frac{\partial p^{p}}{\partial t} \\
& v^{p}-\phi^{p} \frac{\partial u_{i}^{s}}{\partial t}=-\frac{K}{\mu} \frac{\partial p^{p}}{\partial x_{i}}
\end{aligned}
$$

where: $\sigma^{m}$ is the total stress, $\sigma^{s}$ is the solid partial stress, $p^{p}$ is the water pore pressure in the microporous matrix, $v^{p}$ is the average pore water velocity, $u^{s}$ is the displacement, $e_{x}\left(\boldsymbol{u}^{s}\right)$ is the deformation tensor, $\boldsymbol{c}$ is the elastic tensor, $\phi^{p}$ is the porosity in the microporosity domain, $\mu$ is water dynamic viscosity and $\boldsymbol{x}$ is the non- 
dimensional space variable $(\boldsymbol{x}=\boldsymbol{X} / L)$. For simplicity, the Biot tensor $\boldsymbol{\alpha}$ and the conductivity tensor $\boldsymbol{K}$ are assumed isotropic.

\section{In the crack (vug) domain:}

$$
\begin{aligned}
& \frac{\partial \sigma_{i j}^{c}}{\partial x_{j}}=0 \\
& \sigma_{i j}^{c}=2 \varepsilon^{4} \mu e_{x i j}\left(v^{c}\right)-p^{c} I_{i j} \\
& \frac{\partial v_{i}^{c}}{\partial x_{i}}=0
\end{aligned}
$$

where: $v^{c}$ is the fluid velocity, $p^{c}$ is the water pressure in the crack (vug), $e_{x}\left(v^{c}\right)$ is the rate of deformation tensor, $\varepsilon<<1$ is the homogenization parameter. The coefficient $\varepsilon^{4}$ in Equation (6) results from estimations of orders of magnitude of different terms appearing in the description (Lewandowska and Auriault, 2012):

\section{At the microporous-crack (vug) interface:}

$$
\begin{aligned}
& \sigma_{i j}^{m} n_{j}=\sigma_{i j}^{c} n_{j} \\
& p^{p}=p^{c} \\
& \left(v^{p}-\phi^{p} \frac{\partial u_{i}^{s}}{\partial t}\right)+\frac{\partial u_{i}^{s}}{\partial t}=v^{c}
\end{aligned}
$$

where: $\boldsymbol{n}$ is the unit normal vector which is exterior to $\Omega_{m}$.

\section{MACROSCOPIC PROBLEM}

By following the asymptotic expansions method (Sanchez-Palencia, 1980), we look for the unknown quantities in the form

$\varphi=\varphi^{(0)}(x, y, t)+\varepsilon \varphi^{(1)}(x, y, t)+\varepsilon \varphi^{(2)}(x, y, t)+$.

where $y=x / \varepsilon$ and all the terms $\varphi^{(i)}(x, y, t)$ are y-periodic. Then, we introduce the expansions (11) into the problem (1)-(10) and equal the terms appearing at successive orders of $\varepsilon$. After the analysis of each problem we obtain the macroscopic description in the form (12)-(18) (Lewandowska \& Auriault, 2012]:

$$
\frac{\partial \sigma_{i j}^{M}}{\partial x_{j}}=0
$$


where $\sigma_{i j}^{M}=c_{i j k l}^{e f f} e_{x k l}\left(\boldsymbol{u}^{s(0)}\right)-\alpha_{i j}^{e f f} p^{(0)}$ are the components of the total macroscopic stress. The macroscopic tensors $c_{i j k l}^{\text {eff }}$ and $\alpha_{i j}^{e f f}$ are defined as the volume averages over the period as follows:

$$
\begin{aligned}
& c_{i j k l}^{e f f}=\left\langle c_{i j k l}+c_{i j m n} e_{y m n}\left(\xi^{k l}\right)\right\rangle \\
& \alpha_{i j}^{e f f}=\left(\varphi^{c}+\left(1-\varphi^{c}\right) \alpha\right) I_{i j}+\left\langle(1-\alpha) c_{i j k l} e_{y k l}(\eta)\right\rangle
\end{aligned}
$$

$\varphi^{c}$ is the volumetric fraction of cracks (vugs) in the period. The symbol <. > stands for the volume average.

The macroscopic Darcy law is derived in the form:

$$
v_{i}^{M(0)}=-\frac{K_{i j}^{e f f}}{\mu} \frac{\partial p^{(0)}}{\partial x_{j}}
$$

where the macroscopic water conductivity is defined as the surface average

$$
K_{i j}^{e f f}=\frac{1}{|\Omega|} \int_{\partial \Omega} \frac{K}{\mu} y_{i}\left(I_{j l}-\frac{\partial \tau_{j}}{\partial y_{l}}\right) n_{l} d S
$$

Finally, the macroscopic mass balance equation takes the form:

$$
\frac{\partial}{\partial x_{i}}\left(v^{M(0)}\right)=-\alpha_{u j}^{e f f} \frac{\partial e_{\mathrm{xij}}\left(\boldsymbol{u}^{s(0)}\right)}{\partial t}-\beta^{e f f} \frac{\partial p^{(0)}}{\partial t}
$$

where the second macroscopic Biot parameter $\beta^{\text {eff }}$ is given by Eq. (19) (after correction of the exponent of $(1-\alpha))$

$$
\beta^{e f f}=\left(1-\varphi^{c}\right) \beta+(1-\alpha)\left\langle e_{y i i}(\eta)\right\rangle
$$

Note that the vectors $\xi, \eta$ and $\tau$ are solutions of local boundary value problems in the period (see Lewandowska \& Auriault, 2012).

It can be seen that the macroscopic problem (11)-(18) presents the same form as the classical Biot model. However, the macroscopic parameters are modified due to the damage of the porous medium. 


\section{NUMERICAL EXAMPLE}

Let us consider a periodic porous medium with a period $1 \times 1$ which contains circular vug of diameter 0.4 in the center. The local (mesoscopic) parameters of the porous medium are: Young modulus $\mathrm{E}=1 \mathrm{GPa}$, Poisson ratio $v=0.3$, porosity $\mathrm{n}=0.126$, Biot parameters: $\alpha=0.334$ and $\beta=0.218 \mathrm{GPa}^{-1}$. The numerical solutions of the local boundary value problems (see Lewandowska \& Auriault, 2012) give the following values of the macroscopic Biot parameters of the damaged porous medium: $\alpha^{\text {eff }}=0.556$ and $\beta^{\text {eff }}=0.336$.

\section{Macroscopic boundary value problem}

We solve a macroscopic boundary value problem for the geometry presented in Figure 2. The example was inspired by water pumping problem solved in (Leak and Hsiek, 1997). Our example concerns water injection into the layer 2 . The aim of the computations is to investigate the influence of the parameters of the layer 2 for which two sets of parameters were assumed: i) case 1: without damage (without vugs) and ii) case 2: with damage (with vugs). The layers 1 and 3 have the same mechanical and hydraulic parameters. All data are collected in Table 1.



Figure 2. Definition of the macroscopic problem.

The extended Biot problem (Equations 13-16-18) was solved in domain ABCDEFGHIJ with the following boundary conditions:

\section{Hydraulic problem:}

- Impervious boundary: DE, EF, FG, BC, GH, HI.

- Constant hydraulic charge $\mathrm{H}=0: \mathrm{AB}, \mathrm{AJ}, \mathrm{IJ}$.

- Time dependent hydraulic charge: $H(t)=+6(\mathrm{~m} /$ year $) \times t$ 


\section{Mechanical problem:}

- Zero displacement boundary $\mathrm{u}=\mathrm{v}=0$ : DE, EF, FG.

- Free of charge boundary: AJ

- Symmetry condition $\mathrm{u}=0$ : AB, BC, CD, GH, HI, IJ.

Table 1. Physical parameters of the layers.

\begin{tabular}{|l|c|c|}
\hline \multicolumn{1}{|c|}{ Quantity } & Layers 1 and 3 & Layer 2 \\
\hline & & \\
\hline Hydraulic conductivity & $2.89 \times 10^{-4} \mathrm{~m} / \mathrm{s}$ & $1.16 \times 10^{-7} \mathrm{~m} / \mathrm{s}$ \\
Young modulus & $800 \mathrm{MPa}$ & $80 \mathrm{MPa}$ \\
Poisson ratio & 0.25 & 0.25 \\
Biot parameters & $\alpha=1$ & Case $1:$ \\
& $\beta=1.02 \times 10^{-10} \mathrm{~Pa}^{-1}$ & $\alpha=0.334$ \\
& & $\beta=2.73 \times 10^{-9} \mathrm{~Pa}^{-1}$ \\
& & Case $2:$ \\
& & $\alpha=0.556$ \\
& & $\beta=4.12 \times 10^{-9} \mathrm{~Pa}^{-1}$ \\
\hline
\end{tabular}

\section{Results}

The problem was solved twice (Case 1 and Case 2). In Figure 3 hydraulic head, velocity streamlines and mechanical deformation after water injection for Case 2 , are shown. A similar general behaviour is observed in Case 1. We can see ground heave (vertical displacement in the upward direction) at the surface.

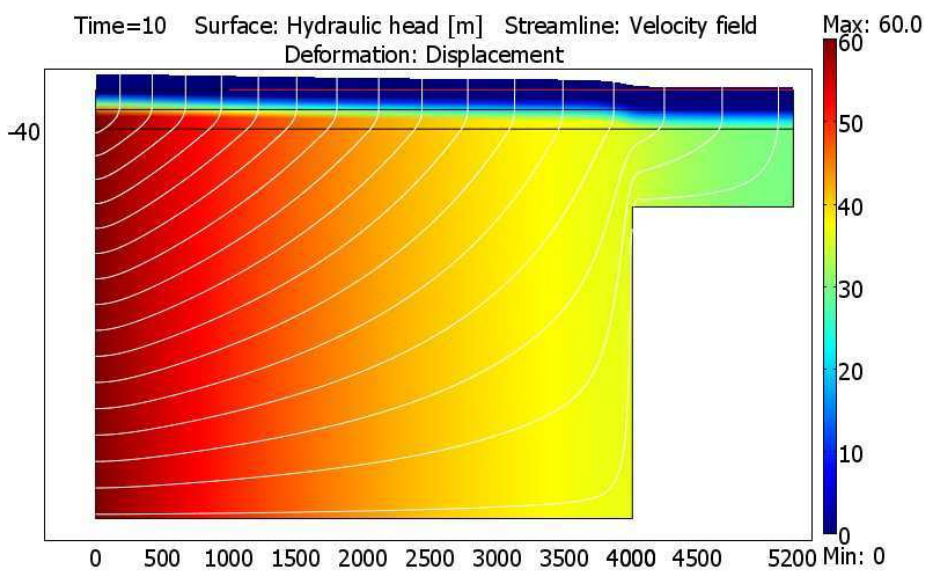

Figure 3. Hydraulic head, velocity streamlines and mechanical deformation after water injection for Case 2. 
Figure 4 shows the heave of the ground surface at different time in two cases in Cases 1 and 2. As expected, Case 2 leads to higher values of the heave with respect to Case 1. It proves that material damage is responsible for greater deformations.

a)

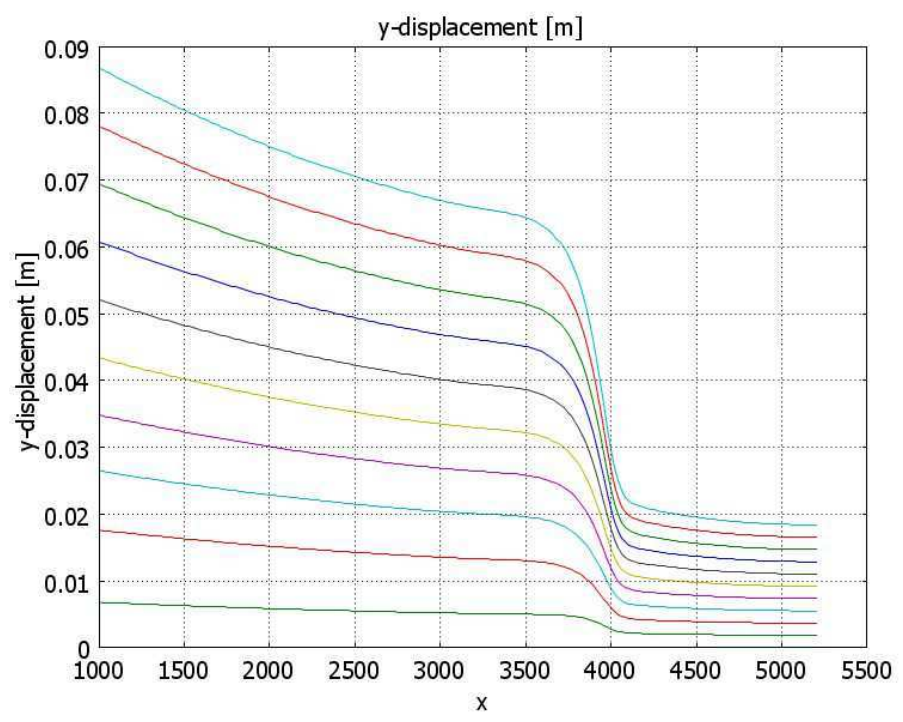

b)

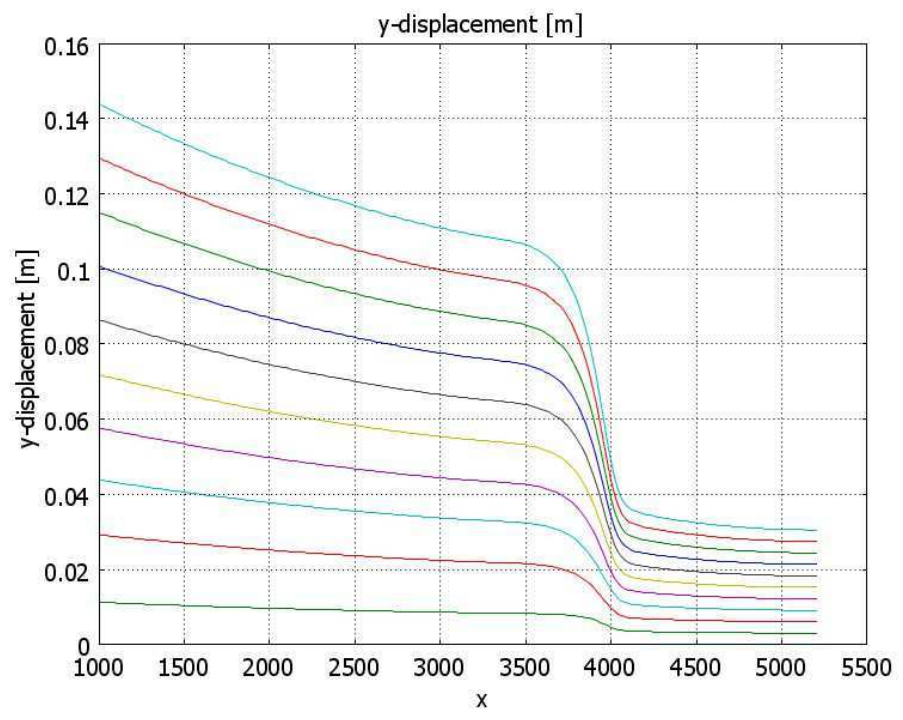

Figure 4. Heave of the ground surface for different times (from 1 to 10 years): a) Case 1, b) Case 2.

In Figure 5 strains in the horizontal direction at the soil surface for different times (from 1 to 10 years) in Case 1 and Case 2, are presented. It can be seen that the maximum extension (positive) as well as the maximum compression strains (negative) are greater in Case 2 than in Case 1. Even though the volumetric fraction 
of cracks (vugs) is only 0.126 , there is a factor 2 between the maximum values of strains. It clearly demonstrates the impact of damage of porous material on its hydromechanical behaviour.

a)

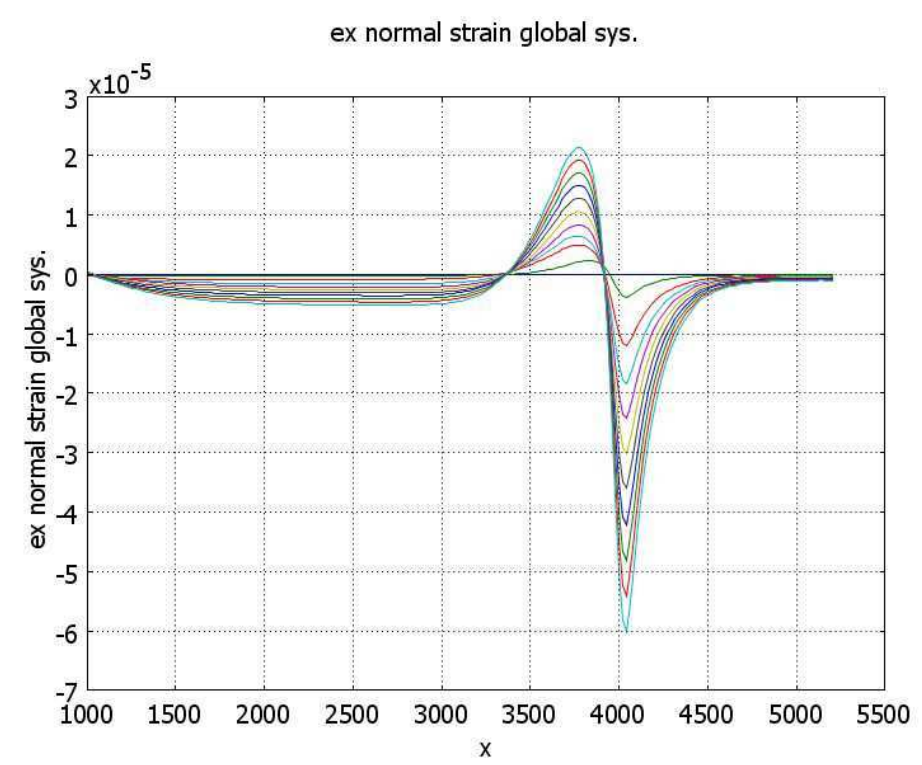

b)

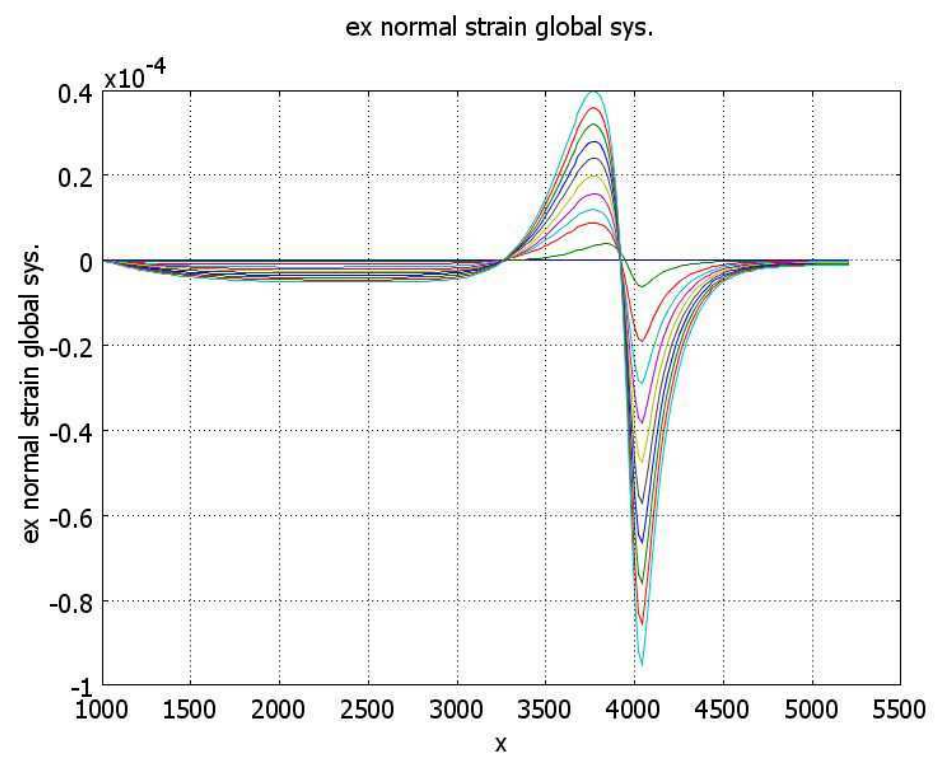

Figure 5. Strains in the horizontal direction at the soil surface at different times (from 1 to 10 years): a) Case 1, b) Case 2. 


\section{CONCLUSIONS}

This paper presents the extension of the Biot model to the case of damaged porous material, containing isolated cracks or/and vugs. The model was obtained by using the method of asymptotic expansions. It was shown that the corrections of effective parameters of the Biot original model are necessary. The numerical computations of a particular boundary value problem show the quantitative influence of cracks (vugs) present in the microstructure on the macroscopic hydro-mechanical behaviour of the medium.

\section{REFERENCES}

Biot, M. A. (1941). "General theory for three-dimensional consolidation." J. of Applied Physics, 12, 155-164.

Leak S.A. and Hsiek P.A. (1997). "Simulation of deformation of sediments from decline of ground-water levels in an aquifer underlain by a bedrock step". US Geological Survey, Open file report, 47-97.

Lewandowska, J. and Auriault J.-L. (2012). "Extension of Biot theory to the problem of saturated microporous elastic media with isolated cracks or/and vugs." Int. J. for Numer. Anal. Meth. Geomech., DOI:10.1002/nag.2150.

Sanchez-Palencia, E. (1980). "Non-homogeneous Media and Vibration Theory." Springer-Verlag, Berlin. 